\title{
BRIEF
}

\section{Evaluation of a Rubric to Assess Pharmacy Student Performance in a Telehealth Simulation Exercise}

\author{
Michelle N. Schroeder, PharmD, Aaron J. Lengel, PharmD \\ University of Toledo, College of Pharmacy \& Pharmaceutical Sciences, Toledo, Ohio
}

Corresponding Author: Michelle N. Schroeder, University of Toledo, College of Pharmacy \& Pharmaceutical Sciences. 3000 Arlington Ave., Toledo, OH 43614. Tel: 419-383-1908. Email: Michelle.mangan@utoledo.edu

Submitted June 17, 2021; accepted October 8, 2021; ePublished October 2021

Objective. To evaluate a telehealth simulation rubric for a pharmacy skills lab course using inter-rater reliability (IRR) comparing different evaluator types.

Methods. A rubric was developed and used to score student pharmacists' competency in a telehealth simulation for a pharmacy skills lab course. Intra-class correlation coefficients (ICC) were calculated to determine the IRR.

Results. Fifty-five recorded telehealth simulation sessions were scored three times by a pool of evaluators using the rubric. The overall ICC for the rubric was 0.631 across different types of evaluators, indicating good IRR. Differences in ICC were observed when analyzed by type of evaluator and rubric sub-components.

Conclusion. The rubric demonstrated good IRR across multiple types of evaluators when used in a skills lab course. Additional evaluation of the rubric and continuous quality improvement is warranted to ensure that assessment becomes more consistent with continued use, regardless of the evaluator type.

Keywords: telehealth, telepharmacy, tele-education, etiquette, rubric

\section{INTRODUCTION}

The utilization of telehealth services increased during the COVID-19 pandemic as an essential way to provide continued health care access amidst stay-at-home orders and social distancing protocols. Telehealth visits increased by 50 percent in March 2020, compared to the same period in 2019, and more than one in eight Americans had engaged in a video consult with a health care provider at that time. ${ }^{1}$ Temporary changes to federal law and regulations implemented early in the pandemic increased access and created further opportunities for pharmacists to receive reimbursement for telehealth services. ${ }^{2}$ Telehealth is a broad term that refers to using electronic information and telecommunications technologies to support and promote long-distance care, monitoring, and education. ${ }^{3}$ In the pharmacy setting, telehealth may be referred to as tele-pharmacy which is specific to pharmacy care provided to patients through telecommunications. ${ }^{4}$ Telehealth services provided by pharmacists include medication therapy management, chronic disease state management, remote order entry, medication dispensing, patient education, transitions of care, and others. ${ }^{5}$ Early on in the pandemic, pharmacists and other health professionals had to make the quick transition to delivering telehealth services to ensure continuity of care.

The global pandemic also highlighted opportunities for pharmacy educators to evaluate the teaching of timely topics like tele-pharmacy and telehealth. It has become apparent that the increase in the use of telehealth accompanied by regulatory changes is likely to lead to continued use of these services after the pandemic. ${ }^{6}$ Therefore, pharmacy students will need training on various aspects important to the delivery of telehealth services. ${ }^{7}$ Literature published on telehealth and pharmacy students describes simulation activities and student perceptions on how a virtual environment affects understanding, confidence, communication, and abilities in interprofessional collaboration. ${ }^{8-12}$ Student performance scores on virtual skills-based assessments compared to in-person have also been published, but a method for evaluating competency of skills performed in a telehealth activity that can be used among different evaluator types is not currently available. ${ }^{12-14}$

Telehealth etiquette refers to unique behaviors outside of typical professional actions that are used to conduct an effective telehealth visit. ${ }^{15}$ Both verbal and non-verbal communication skills are components of telehealth etiquette that lead to increased patient satisfaction and successful interactions. ${ }^{16-17}$ Timing and pace of a telehealth visit as well as the use of motivational interviewing are considerations to help improve verbal communication. ${ }^{18}$ Eye contact is a non-verbal technique that is more challenging to accomplish via telehealth. Looking at the camera rather than at the patient on the screen will help the provider to give the appearance of making direct eye contact. In a virtual setting, health professionals will need to develop the patient-professional relationship differently and more deliberately than an in-person encounter. ${ }^{19}$ 
If not addressed appropriately, personal appearance, environmental factors, privacy, and problem-solving skills could significantly impact the quality of a telehealth encounter compared to an in-person visit. ${ }^{15}$ Personal appearance during a virtual visit can be more affected by clothing color, pattern choices, or jewelry selection. ${ }^{19}$ Workspace considerations that can improve the environment include minimizing background noise, ensuring proper lighting, and determining what is viewable behind you. Privacy and security are also necessary and can be of concern for patients when they cannot see the virtual environment is secure. Communicating that the area is secure, introducing all individuals present, and using the camera to show the patient around the room are ways to minimize these concerns. ${ }^{12}$ Lastly, problem-solving skills are necessary to help overcome potential technological barriers and to manage any unforeseen factors that may impact the effectiveness of a telehealth session. The primary goal of the following study was to evaluate a telehealth simulation rubric using inter-rater reliability (IRR).

\section{METHODS}

A six-semester pharmacy skills development (PSD) laboratory course is part of the professional Doctor of Pharmacy curriculum at the University of Toledo. Sixty-seven third-year pharmacy students were enrolled in the fifth PSD course of the series (PSD-5) during Fall 2020. A one-hour lecture was given to students as an introduction to the topic of telehealth. The lecture included telehealth definitions, recent regulatory changes, reimbursement, privacy and confidentiality, audio and visual technologies, and communication best practices/etiquette. At that time, the lecture component of the course was delivered remotely.

Following the lecture, students participated in a telehealth simulation as an in-lab graded activity where they met with a mock patient using Blackboard Collaborate. The virtual platform had audio and video capabilities, and all participants were expected to have their microphones and cameras on during the session. Lab was conducted remotely, and participants were primarily connecting from home. Students were scheduled in twenty-minute time blocks to participate in the simulation. Sessions were recorded following normal course practice in the case of grading discrepancies. The purpose of the session was to collect a medication history from a patient recently discharged from hospital to home and to reconcile discrepancies identified in real-time. A patient scenario and medication list, medication history interview handout, and documentation template were provided to the students prior to the session to assist with preparation.

The role of the patient was fulfilled using volunteer faculty $(n=2)$, residents $(n=6)$, and one advanced pharmacy practice experience (APPE) student completing an elective academia rotation. A mock patient scenario, including background, patient information, and current medication knowledge from the patient's perspective, was provided prior to the activity to standardize the interaction and patient responses. The person playing the mock patient also served as the evaluator. Student performance was scored using a competency and communication rubric from the course series that was modified to include statements to assess telehealth etiquette skills. The coordinator of the course determined which telehealth etiquette skills would be included in the rubric based on practice experience and a review of the literature as outlined above.

The rubric used for the study contained 13 assessment statements for competency and six for telehealth etiquette (Figure 1). Each statement was evaluated based on three dimensions, satisfactory, unsatisfactory, or not performed. The rubric did not describe criteria for any of the dimensions beyond the assessment statements. Students were provided the rubric one week prior to participating in the simulation. A brief overview of the activity scenario and the rubric was provided to evaluators before the session, but no specific training or guidance was provided on how to interpret the rubric. Upon activity completion, the completed rubric containing quantitative and qualitative feedback was provided to the student.

Upon semester completion, two additional faculty evaluators reviewed the recorded sessions and evaluated the students using the rubric to gather retrospective data on inter-rater reliability (IRR). The intended purpose of using IRR for this study was to assess reliability of the mean ratings from multiple coders. Of 67 students in the class, 55 recordings were able to be reviewed a total of three times each. Seven sessions were evaluated by three faculty members, 36 sessions were evaluated by a resident and two faculty, and 12 sessions were evaluated by the APPE student and two faculty. There were four missing recordings and eight incomplete recordings that were excluded from the sample. The intra-class correlation (ICC) coefficient was calculated to determine the IRR of the rubric overall and its competency and telehealth etiquette sub-components. A $p$ value of less than .05 was considered significant. A one-way random effect model and average measures mean (ICC $[1,3]$ ) was used to calculate ICC when the evaluators for each student were not the same three evaluators each time. A two-way mixed effect model and average measures mean (ICC [3,3]) was used to calculate ICC when the same three evaluators reviewed a recording. Higher ICC values indicated stronger IRR, which was suggestive of stronger consistency among evaluators. The ICC scores less than 0.4 indicated poor IRR, scores between 0.4-0.59 indicated fair IRR, scores between 0.6 and 0.74 indicated good IRR, and scores between 0.75 and 1 indicated 
excellent IRR. ${ }^{20}$ Statistical analysis was performed using SPSS Statistics, version 27 (IBM Corp; Armonk, NY). This study was reviewed by the University of Toledo Social, Behavioral, and Educational Institutional Review Board and was exempted as not human subjects research.

\section{RESULTS}

The ICC results for this study representing IRR are presented in Table 1. The ICC when including all evaluators in the pool was $0.631(p<.001 ; 95 \%$ CI, 0.423-0.772), indicating good IRR for the rubric overall. Calculating ICC for the sessions evaluated only by faculty members as the three evaluators showed the strongest consistency among evaluator types. IRR diminished compared to faculty when residents or a student were a part of the evaluator pool.

ICC for the 13 competency statements of the rubric was assessed and compared among evaluator types. The ICC for the competency statements of the rubric was $0.691(p<.001 ; 95 \%$ CI $0.517-0.809)$ for all evaluators indicating good IRR. Excellent IRR was shown for the competency statements in the rubric evaluated by faculty when calculating ICC. Again, IRR diminished for the competency component of the rubric when the evaluator group included residents.

Comparatively, the ICC for all evaluators for the six telehealth etiquette statements of the rubric was 0.468 ( $p=.003 ; 95 \%$ CI, 0.169-0.672), indicating fair IRR. The ICC for the telehealth etiquette statements of the rubric suggested fair IRR among faculty evaluators but was not statistically significant. The ICC for the telehealth etiquette statements of the rubric for the resident-faculty evaluator group indicated fair IRR. The ICC for the telehealth etiquette statements suggested poor IRR when including a student as an evaluator but was not statistically significant.

\section{DISCUSSION}

In a recent commentary, Frenzel and Porter clearly described the relevance and need to prepare pharmacy students to be able deliver services remotely. ${ }^{7}$ While descriptions of telehealth simulation activities have been published, a method to evaluate telehealth etiquette skills that can be used among varying evaluator types is not available. ${ }^{8,10,12,14}$ Candelario and colleagues described a telemedicine simulation where pharmacy students worked in a group to identify medicationrelated problems (MRPs) incorporated into a discharge follow-up encounter. Standardized patients completed a patient perception scale checklist to assess communication skills. Less than half of the student teams performed communication skills for this activity accurately and completely. ${ }^{8}$ Although methods to assess student performance in a telehealth simulation were discussed, this study focused more on transitions of care concepts and did not mention assessment of telehealth etiquette skills for the activity. Gustin and colleagues presented a study which described a training program developed to teach health care students telehealth etiquette skills. A telehealth etiquette checklist was used to help facilitate discussion, reflection, and feedback on techniques but was not utilized as an assessment tool. ${ }^{12}$

The IRR of the telehealth etiquette statements for the rubric used in this study was not consistent across evaluator types which indicates that improvement efforts should be focused on this area. In addition to potential revisions to rubric statements, a description of expectations among the different dimensions is needed to improve the clarity of the rubric overall. Telehealth may also have been a new concept to the evaluators and something they may not have had enough personal practice experience in. While not recorded in this study, the professional background of a rater could also affect rater reliability considering raters may not have had the same types of experiences to draw upon when completing the rubric. Another factor impacting IRR was that there was no formal training in interpreting the telehealth statements provided to evaluators before participating in the activity. To help standardize scoring and improve consistency among evaluators, training will include instruction on how to evaluate student performance, including a discussion of the rubric, and to have the opportunity to assess a sample session using the rubric to model.

The current study demonstrated good IRR across different evaluator types, which also supports the need for continued development of the assessment tool. The IRR of the rubric was highest when faculty members used the tool to evaluate students, but this may not be a realistic recommendation to make considering faculty workloads and responsibilities. Allowing residents and students to assist in the simulation and evaluation process is also valuable to their development as potential educators. Of note, the student-faculty evaluator group had a higher ICC for the rubric compared to the resident-faculty evaluator group, but this is likely due to the student assisting with developing some rubric statements as part of a rotation project. When reviewing the IRR results of the competency assessment statements in the rubric, fair to good IRR was generally seen across all groups except for the faculty-only group, which showed excellent IRR. This is likely because the faculty evaluators have used the competency and communication portion of the rubric in other skills lab courses. The competency statements had already been standardized for the course series and originally developed with assistance from the faculty involved in this research. Utilizing external faculty for further evaluation of the rubric will be important in expanding IRR results.

There are limitations to take into consideration for this study. First is that these results capture data from the first use of the rubric among a limited pool of evaluators for one cohort of students. Continued use of the rubric with additional 
evaluators may improve reliability of the rubric over time. Second, the original evaluator of each session was potentially multitasking acting both as the mock patient and evaluating the student in real-time. The first evaluator completed the rubric after each session and did not have the recording to view whereas the evaluators for the study were watching the recordings which may have introduced some bias. Lastly, validation of this rubric is needed before being used in a highstakes assessment versus a formative assessment.

\section{CONCLUSION}

There is not currently a validated rubric available to assess students in telehealth etiquette skills and additional research is needed before the rubric described in this study can be universally implemented in pharmacy education. The rubric applied in this study demonstrated good IRR overall among various evaluator types when used in a skills lab course. Evaluation and quality improvement of the telehealth etiquette statements of the rubric are necessary to aid in improving feedback provided to students on their performance. An evaluator training session on interpreting and using the rubric is also planned to help improve reliability with future assessments.

\section{ACKNOWLEDGEMENTS}

The authors would like to acknowledge the evaluators who assisted with the simulation and initial evaluations. We would also like to thank Dr. Tian Chen for providing statistics consulting and Dr. Mariann Churchwell for her guidance in this research.

\section{REFERENCES}

1. Boner, L. Telehealth basics for pharmacists during COVID-19 and beyond. Pharmacy Today. 2020;26(6):28-29. doi:10.1016/j.ptdy.2020.05.013.

2. Issue Brief: COVID-19 and Telehealth Changes. American Society of Health-System Pharmacists. https://www.ashp.org/Advocacy-and-Issues/Key-Issues/Other-Issues/Issue-Brief-COVID-19. Published April 9, 2020. Accessed June 2, 2021.

3. What is telehealth? U.S Department of Health \& Human Services. https://www.hhs.gov/hipaa/forprofessionals/faq/3015/what-is-telehealth/index.html. Updated March 27, 2020. Accessed June 2, 2021.

4. Alexander E, Butler CD, Darr A, et al. ASHP Statement on Telepharmacy. Am J Health Syst Pharm. 2017;74(9):e236-e241. doi:10.2146/ajhp170039.

5. What is Telehealth? American Pharmacists Association. https://www.pharmacist.com/Practice/PracticeResources/Telehealth. Accessed June 2, 2021.

6. Koonin LM, Hoots B, Tsang CA, et al. Trends in the Use of Telehealth During the Emergence of the COVID-19 Pandemic - United States, January-March 2020. MMWR Morb Mortal Wekly Rep. 2020;69(43):1595-1599. doi:10.15585/mmwr.mm6943a3.

7. Frenzel J, Porter A. Preparing Graduates for Telepharmacy and Telehealth: The Need for Tele-Education. [published ahead of print February 2021]. Am J Pharm Educ. 2021;85(5). doi:10.5688/ajpe8566.

8. Candelario D, Cunningham K, Solano LA, Pabst A, Srivastava S. Description of a transitions of care and telemedicine simulation lab activity. Curr Pharm Teach Learn. 2019;11(11):1184-1198. doi:10.1016/j.cptl.2019.7.017,

9. Almodovar AS, Chang HK, Matsunami M, Coleman A, Nahata MC. Confidence in skills applied to patient care among PharmD students in telehealth medication management programs versus other settings. Curr Pharm Teach Learn. 2018;10(5):558-565. doi:10.1016/j.cpt1.2018.02.008.

10. Begley K, O’Brien K, Packard K, et al. Impact of Interprofessional Telehealth Case Activities on Students' Perceptions of Their Collaborative Care Abilities. Am J Pharm Educ. 2019;83(4):6880. doi:10.5688/ajpe6880.

11. Beal JL, Weber ZA, Isaacs AN, Illingworth Plake KS, Zillich A, Woodyard JL. Pharmacy Student Perceptions and Preferences of In-person Versus Video Recorded Evaluations in Skills-Based Courses. Am J Pharm Educ. 2020;84(11):7976. doi:10.5688/ajpe7976.

12. Gustin TS, Kott K, Rutledge C. Telehealth Etiquette Training: A Guideline for Preparing Interprofessional Teams for Successful Encounters. Nurse Educ. 2020:45(2);88-92. doi:10.1097/nne.0000000000000680.

13. Skoy ET, Eukel HN, Frenzel JE, Schmitz TM. Performance and Perceptions: Evaluation of Pharmacy Students' Consultation via Telepharmacy. J Pharm Technol. 2015;31(4):155-160. doi:10.117//87551225145681123.

14. VanLangen KM, Sahr MJ, Salvati LA, Meny LM, Bright DR, Sohn M. Viability of Virtual Skills-based Assessments Focused on Communication. [published ahead of print January 2021]. Am J Pharm Educ. 2021;8378. doi:10.5688/ajpe8378. 
15. Haney T, Kott K, Fowler C. Telehealth etiquette in home healthcare: the key to a successful visit. Home Healthc Now. 2015;33(5):254-259. doi:10.1097/NHH. 0000000000000228.

16. Bulik RJ. Human factors in primary care telemedicine encounters. J Telemed Telecare. 2008;14(4):169-172. doi:10.1258/jtt.2007.007041.

17. Ben-Arieh D, Charness N, Duckett K, Krupinski E, Leistner G, Strawderman L. A Concise Guide for Telemedicine Practitioners: Human Factors Quick Guide - Eye Contact. American Telemedicine Association. https://www.telemedecine-360.com/wp-content/uploads/2019/02/2016-ATA-Eye-Contact-QuickGuide.final_.pdf. Published February 2016. Accessed June 2, 2021.

18. Rienits H, Teuss G, Bonney A. Teaching telehealth consulting skills. Clin Teach. 2016;13(2):119-123. doi:10.1111/tct.12378.

19. Iafolla T. 10 Telemedicine Etiquette Tips to Deliver Professional Care. eVisit. https://blog.evisit.com/10telemedicine-etiquette-tips-deliver-professional-care. Published December 21, 2015. Accessed June 2, 2021.

20. Cicchetti DV. Guidelines, Criteria, and Rules of Thumb for Evaluating Normed and Standardized Assessment Instruments in Psychology. Psychol Assess. 1994;6(4):284-290. doi:10.1037/1040-3590.6.4.284.

Table 1. Intraclass Correlation Coefficient Results for Inter-rater Reliability

\begin{tabular}{|c|c|c|c|c|c|}
\hline Item & $\begin{array}{c}\text { Number of } \\
\text { sessions }\end{array}$ & ICC & IRR & $95 \% \mathrm{CI}$ & $p$ value \\
\hline \multicolumn{6}{|l|}{ All evaluators } \\
\hline Entire rubric & & 0.631 & Good & $0.423-0.772$ & $<.001$ \\
\hline Competency & 55 & 0.691 & Good & $0.517-0.809$ & $<.001$ \\
\hline Etiquette & & 0.468 & Fair & $0.169-0.672$ & .003 \\
\hline \multicolumn{6}{|c|}{ Faculty evaluators only } \\
\hline Entire rubric & & 0.881 & Excellent & $0.555-0.978$ & .001 \\
\hline Competency & 7 & 0.934 & Excellent & $0.754-0.988$ & $<.001$ \\
\hline Etiquette & & 0.571 & Fair & $-0.598-0.920$ & .100 \\
\hline \multicolumn{6}{|c|}{ Residents \& faculty evaluators } \\
\hline Entire rubric & & 0.538 & Fair & $0.199-0.748$ & .003 \\
\hline Competency & 36 & 0.571 & Fair & $0.257-0.766$ & .001 \\
\hline Etiquette & & 0.589 & Fair & $0.288-0.776$ & $<.001$ \\
\hline \multicolumn{6}{|c|}{ Student \& faculty evaluators } \\
\hline Entire rubric & & 0.670 & Good & $0.127-0.897$ & .013 \\
\hline Competency & 12 & 0.751 & Excellent & $0.342-0.922$ & .003 \\
\hline Etiquette & & 0.372 & Poor & $-0.662-0.804$ & .170 \\
\hline
\end{tabular}

$\mathrm{ICC}=$ intra-class correlation coefficients; $\mathrm{IRR}=$ inter-rater reliability; $\mathrm{CI}=$ confidence interval 


\begin{tabular}{|l|}
\hline Professional Competence \& Communication Ability \\
\hline Identifies/introduces self \& role. \\
\hline Explains purpose \& structure of the session, including estimated duration. \\
\hline Identifies/confirms patient or patient's agent using two identifiers. \\
\hline Verifies the patient's current pharmacy/pharmacies used. \\
\hline Assesses for suspected or documented drug allergies/sensitivities/intolerances AND reactions. \\
\hline $\begin{array}{l}\text { Collects detailed medication history (name, strength, dosage form, route of administration, frequency, } \\
\text { indication) for: }\end{array}$ \\
\hline$\quad$ Rx medications \\
- OTC/non-Rx medications \\
- Supplements (vitamins, minerals, herbals) \\
\hline Assesses patient adherence to medication regimen (frequency \& reasoning, last dose taken). \\
\hline Assesses patient understanding of their medication regimen (indication, how to take, etc.). \\
\hline Uses balance of open- and closed-ended questions to assess the patient's actual use of medications. \\
\hline Uses language the patient is likely to understand. Defines any medical terminology used. \\
\hline $\begin{array}{l}\text { Closes the interview appropriately. Allows opportunity for questions. Discusses the need for follow-up as } \\
\text { indicated. }\end{array}$ \\
\hline Telehealth Etiquette \\
\hline Displays problem-solving skills to help overcome technological \& environmental barriers. \\
\hline $\begin{array}{l}\text { Workspace \& Attire portrays professional \& non-distracting environment (i.e. appearance, noise, lighting, } \\
\text { background, HIPAA, etc.). }\end{array}$ \\
\hline Displays planning \& preparedness to run session effectively (both pre- \& during session). \\
\hline Attempts to make meaningful “eye contact" with camera (vs. screen). \\
\hline Pace, tone, volume appropriate enough to communicate clearly. \\
\hline Displays appropriate non-verbal behaviors (facial expression, gestures, posture, etc.). \\
aAll statements evaluated using Satisfactory (1 point), Unsatisfactory (0.5 points), or Not Performed (0 points) \\
\hline
\end{tabular}

${ }^{a}$ All statements evaluated using Satisfactory (1 point), Unsatisfactory ( 0.5 points), or Not Performed ( 0 points) 